\title{
Shape resonances in the photoionization of $\mathrm{N}_{\mathbf{2}} \mathrm{O}$
}

\author{
M. Braunstein and V. McKoy \\ Arthur Amos Noyes Laboratory of Chemical Physics, ${ }^{\text {) }}$ California Institute of Technology, Pasadena, California \\ 91125
}

(Received 12 February 1987; accepted 25 March 1987)

\begin{abstract}
We report the results of studies of the cross sections and photoelectron asymmetry parameters for photoionization of the $7 \sigma$ level of $\mathrm{N}_{2} \mathrm{O}$ using Hartree-Fock photoelectron continuum orbitals. These studies were motivated by recent measurements which showed significant non-Franck-Condon vibrational distributions at low photoelectron energies where previously only autoionizing resonances, but no shape resonance, had been identified. Our results establish that there are two $\sigma$ shape resonances in the $7 \sigma$ ionization continuum, a pronounced resonance at low photoelectron energies, and another at higher energy which is essentially obscured in the vibrationally unresolved cross sections. The shape resonant structure that emerges from these studies differs significantly from the predictions of previous model studies. Studies in progress reveal a rich and unusual dependence of these resonances on changes in internuclear distances.
\end{abstract}

\section{INTRODUCTION}

Shape resonances play a central role in studies of the dynamics of molecular photoionization. These resonances or quasibound states, formed by the trapping of photoelectrons by the centrifugal barrier of the molecular force field, give rise to several distinct features in the photoionization spectra of molecules such as enhancement of the cross sections, a pronounced influence on photoelectron angular distributions, and non-Franck-Condon effects in vibrationally resolved spectra. ${ }^{1}$ Furthermore, shape resonances are being identified in an increasingly wide range of small molecules.

The effects of shape resonances have been studied in many molecules using the intense tunable radiation provided by synchrotron sources. Such experimental studies and related theoretical efforts have served to clarify many of the properties associated with shape resonances such as their positions and symmetries in several diatomic molecules, e.g., $\mathrm{N}_{2}, \mathrm{CO}, \mathrm{O}_{2}$, and $\mathrm{NO}$ and in polyatomic molecules, e.g., $\mathrm{CO}_{2}$, $\mathrm{C}_{2} \mathrm{H}_{2}$, and $\mathrm{C}_{2} \mathrm{~N}_{2}{ }^{2}$

These results have already shown that resonance features in the photoionization of polyatomic molecules, e.g., $\mathrm{CO}_{2}{ }^{3,4}$ and $\mathrm{C}_{2} \mathrm{~N}_{2}{ }^{5,6}$ can behave quite differently from what could be expected on the basis of simple diatomic molecules. For example, although it is uncommon to find two shape resonances in the same continuum channel, studies of the photoionization of cyanogen have identified two such resonances in the $\mathrm{k} \sigma_{u}$ continuum within about $20 \mathrm{eV}$ of photoelectron kinetic energy of each other. ${ }^{7}$ Furthermore, shape resonant vibrational branching ratios and their associated photoelectron angular distributions have been more extensively studied in diatomic molecules, e.g., $\mathrm{N}_{2}^{8}$ and $\mathrm{CO}, 9,10$ than in polyatomic systems such as $\mathrm{CO}_{2}{ }^{11}$ and $\mathrm{N}_{2} \mathrm{O} \cdot{ }^{12-14}$ With their alternative vibrational modes, vibrationally resolved studies of shape resonances in polyatomic molecules can provide rich dynamical insight into molecular photoionization. In many of these polyatomic molecules, shape resonances may result from the interaction between resonances

\footnotetext{
Contribution No. 7545 .
}

associated with subgroups of the molecule. Vibrationally resolved data will be needed in order to understand the behavior of these shape resonances for alternative internuclear configurations and to develop a more intuitive understanding of shape resonances in polyatomic molecules.

From dispersed fluoresence measurements, Poliakoff $e t$ $a l .{ }^{14}$ recently determined the vibrational branching ratios for the symmetric and asymmetric stretching modes in photoionization of the $7 \sigma$ level of $\mathrm{N}_{2} \mathrm{O}$ leading to the $A^{2} \Sigma^{+}$state of $\mathrm{N}_{2} \mathrm{O}^{+}$. The ionization potential of this $7 \sigma$ level is about $16.4 \mathrm{eV}$ and measurements were carried out for photon energies from about 17 to $22 \mathrm{eV}$. The vibrational branching ratios were found to be energy dependent, implying a breakdown of the Franck-Condon approximation due to underlying resonances in this spectral region. Poliakoff et al. ${ }^{14}$ suggested that these results were due to the wings of a shape resonance with autoionizing resonances superimposed in certain narrow spectral subregions. Earlier vibrationally unresolved and resolved photoelectron studies of this channel ${ }^{12.13}$ had identified resonance behavior in this same region. However, in these studies this non-Franck-Condon behavior was generally interpreted in terms of autoionizing resonances. This assignment was partially motivated by the results of multiple scattering model studies of these cross sections which did not show any shape resonance within a few electron volts of the $7 \sigma$ ionization threshold. ${ }^{12}$

We have initiated vibrationally resolved studies of the $7 \sigma$ photoionization of $\mathrm{N}_{2} \mathrm{O}$ so as to provide some further quantitative insight into the effect of shape resonances on the branching ratios and photoelectron angular distributions for the symmetric and asymmetric stretching modes over a wide spectral range. ${ }^{15}$ Here we present the results of these studies at the equilibrium geometry of the ground state. These results reveal an interesting shape resonant structure which, we believe, is perhaps representative of what can be expected in the photoionization spectra of related polyatomic molecules. The results show two shape resonances in the $\sigma$ electronic continuum in $7 \sigma$ photoionization of $\mathrm{N}_{2} \mathrm{O}$. The lowenergy resonance is intense and narrow with a peak cross 
section of about $15 \mathrm{Mb}$ just below $20 \mathrm{eV}$ photon energy or a photoelectron energy of about $3 \mathrm{eV}$. The higher energy shape resonance at a photon energy of about $38 \mathrm{eV}$ is essentially not evident in the vibrationally unresolved cross sections but does lead to a broad, but pronounced, minimum in the photoelectron asymmetry parameter. Both of these resonances can be identified from the behavior of their associated eigenphase sums, illustrating the utility of obtaining eigenphase sums in molecular photoionization studies. The dependence of these eigenphase sums on changes in the $\mathrm{N}-\mathrm{O}$ and $\mathrm{N}-\mathrm{N}$ distances suggest that these resonances cannot be readily associated with a specific region of the molecule. The shifts in the positions of these shape resonances with alternative vibrational modes and their influence on the vibrational branching ratios will be discussed in a future publication. ${ }^{15}$

An outline of the paper is as follows. In Sec. II we briefly discuss the Schwinger variational method which we use to obtain the Hartree-Fock electronic continuum orbitals needed in studies of molecular photoionization and then give some relevant details of the numerical methods used in these studies. In Sec. III our results are presented and compared with available synchrotron radiation data and the results of earlier multiple scattering calculations.

\section{METHOD AND CALCULATIONS}

The rotationally unresolved fixed-nuclei photoionization cross section is given by

$$
\sigma(R)=\frac{4 \pi^{2} \omega}{3 c}\left|\left\langle\Psi_{i}(\mathbf{r}, R)|\mu| \Psi_{f}(\mathbf{r}, R)\right\rangle\right|^{2},
$$

where $\mu$ is the electric dipole operator, $\omega$ is the photon frequency, and $(r, R)$ represents the electronic and nuclear coordinates. In Eq. (1), $\Psi_{i}$ represents the initial state wave function and $\Psi_{f}$ is the wave function for the final ionized state, i.e., molecular ion plus photoelectron. In these studies we use a Hartree-Fock wave function for $\Psi_{i}$. For $\Psi_{f}$ we assume the frozen-core Hartree-Fock model in which the ionic orbitals are constrained to be identical to those of the neutral molecule and the photoelectron orbital is a solution of the one-electron Schrödinger equation

$$
\left[-\frac{1}{2} \nabla^{2}+V_{N-1}(\mathbf{r}, R)-\frac{k^{2}}{2}\right] \phi_{\mathrm{k}}(\mathbf{r}, R)=0,
$$

where $V_{N-1}(r, R)$ is the static-exchange potential of the molecular ion charge density, $k^{2} / 2$ is the photoelectron kinetic energy, and $\phi_{\mathbf{k}}$ satisfies the appropriate scattering boundary conditions.

To solve for $\phi_{\mathbf{k}}$ we work with the integral form of Eq. (2), i.e., the Lippmann-Schwinger equation

$$
\phi_{\mathbf{k}}(\mathbf{r})=\phi_{\mathbf{k}}^{c}+G_{c}^{(-)} V \phi_{k},
$$

where $\phi_{\mathbf{k}}^{c}$ is the Coulomb scattering wave function, $V$ is the molecular ion potential $V_{N-1}$ with the Coulomb potential removed, i.e.,

$$
V=V_{n-1}+\frac{1}{r},
$$

and $G_{c}^{(-)}$is the Coulomb Green's function with incomingwave boundary conditions. Expansion of $\phi_{\mathbf{k}}$ in partial waves, i.e., $\phi_{\mathbf{k}}(\mathbf{r})=\left(\frac{2}{\pi}\right)^{1 / 2} \sum_{l=0} \sum_{m=-l}^{l} i^{i} \psi_{k l m}(\mathbf{r}) Y_{l m}^{*}(\hat{k})$,

and substitution into Eq. (3) shows that each $\psi_{k l m}$ satisfies its own Lippmann-Schwinger equation:

$$
\psi_{k l m}(\mathbf{r})=S_{k l m}+G_{c}^{(-)} V \psi_{k l m} .
$$

We have developed an iterative procedure for solving Eq. (6) which is based on the Schwinger variational method. Details of this technique have been discussed elsewhere ${ }^{17}$ and here we will only outline a few essential features of the procedure. We first obtain an approximate solution to Eq. (6) by assuming a separable approximation to the potential $V$ of Eq. (4) of the form

$$
\begin{aligned}
V\left(\mathbf{r}, \mathbf{r}^{\prime}\right) & \cong V^{s}\left(\mathbf{r}, \mathbf{r}^{\prime}\right) \\
& =\sum_{i, j}\left\langle\mathbf{r}|V| \alpha_{i}\right\rangle\left(V^{-1}\right)_{i j}\left\langle\alpha_{j}|V| \mathbf{r}^{\prime}\right\rangle,
\end{aligned}
$$

where the matrix $V^{-1}$ is the inverse of the matrix with elements $V_{i j}=\left\langle\alpha_{i}|V| \alpha_{j}\right\rangle$ and the $\alpha_{i}$ 's are discrete basis functions such as Cartesian ${ }^{18}$ or spherical ${ }^{19}$ Gaussian functions which are known to be effective in representing the multicenter nature of molecular ion potentials. With this approximation to $V$ in Eq. (6), the solutions of this integral equation can be written as

$$
\begin{aligned}
& \psi_{k l m}^{(0)}(\mathbf{r})=S_{k l m}(\mathbf{r}) \\
& +\sum_{i, j}\left\langle\mathbf{r}\left|G_{c}^{(-)} V\right| \alpha_{i}\right\rangle\left(D^{-1}\right)_{i j}\left\langle\alpha_{j}|V| S_{k l m}\right\rangle,
\end{aligned}
$$

where the matrix $D^{-1}$ is the inverse of the matrix with elements

$$
D_{i j}=\left\langle\alpha_{i}\left|V-V G_{c}^{(-)} V\right| \alpha_{j}\right\rangle .
$$

With adequate basis sets, $\alpha_{i}$ 's, these approximate solutions $\psi_{k l m}^{(0)}$, which satisfy scattering boundary conditions, can provide quantitatively reliable photoionization cross sections. These cross sections can, furthermore, be shown to be variationally stable. ${ }^{20}$ Starting with these solutions $\psi_{\mathrm{klm}}^{(0)}$, converged solutions of Eq. (5) can be obtained through an iterative procedure. ${ }^{17}$

For the ground state of $\mathrm{N}_{2} \mathrm{O}$, with the electronic configuration $1 \sigma^{2} 2 \sigma^{2} 3 \sigma^{2} 4 \sigma^{2} 5 \sigma^{2} 6 \sigma^{2} 1 \pi^{4} 7 \sigma^{2} 2 \pi^{4}$, we used the SCF Slater basis of McLean and Yoshimine. ${ }^{21}$ Calculations were done at the equilibrium geometry with $\mathrm{N}-\mathrm{N}$ and $\mathrm{N}-\mathrm{O}$ bond distances of 2.1273 and 2.2418 a.u., respectively. At this geometry, this basis gives an SCF energy of -183.75668 a.u. For the basis functions, $\alpha_{i}(r)$, in the expansion of Eq. (7) we used spherical Gaussian functions defined by

$$
\chi(\mathbf{r})=N|\mathbf{r}-\mathbf{A}|^{l} \exp \left(-\alpha|\mathbf{r}-\mathbf{A}|^{2}\right) Y_{l m}\left(\Omega_{\mathbf{r}-\mathbf{A}}\right),
$$

where $\mathrm{A}$ locates the origin of the basis function. The basis sets for the $\sigma$ and $\pi$ ionization continua are given in Table $I$.

All matrix elements and functions arising in the solution of Eq. (8) are evaluated using single-center expansions about the central nitrogen atom. The partial wave expansion of the photoelectron orbital, Eq. (5), was truncated at $l_{p}=8$. The other partial wave expansion parameters were chosen as follows: 
TABLE I. Scattering basis sets used in obtaining the photoelectron orbitals."

\begin{tabular}{|c|c|c|c|c|}
\hline Channel & Center & $l$ & $m$ & Exponent \\
\hline$\sigma$ & $\mathbf{N}($ terminal $)$ & $\begin{array}{l}0 \\
1 \\
2\end{array}$ & $\begin{array}{l}0 \\
0 \\
0\end{array}$ & $\begin{array}{l}16,8,4,2,1,0.5 \\
4,2,1,0.5 \\
2,1,0.5\end{array}$ \\
\hline & $\mathbf{N}($ central) & $\begin{array}{l}0 \\
1 \\
2 \\
3 \\
4 \\
5\end{array}$ & $\begin{array}{l}0 \\
0 \\
0 \\
0 \\
0 \\
0\end{array}$ & $\begin{array}{l}16,8,4,2,1,0.5 \\
4,2,1,0.5 \\
2,1,0.5 \\
1 \\
1 \\
1\end{array}$ \\
\hline & 0 & $\begin{array}{l}0 \\
1 \\
2\end{array}$ & $\begin{array}{l}0 \\
0 \\
0\end{array}$ & $\begin{array}{l}16,8,4,2,1,0.5 \\
4,2,1,0.5 \\
2,1,0.5\end{array}$ \\
\hline$\pi$ & $\mathbf{N}($ terminal $)$ & $\begin{array}{l}1 \\
2\end{array}$ & $\begin{array}{l}1 \\
1\end{array}$ & $\begin{array}{l}8,4,2 \\
8,4,2\end{array}$ \\
\hline & $\mathbf{N}$ (central) & $\begin{array}{l}1 \\
2\end{array}$ & $\begin{array}{l}1 \\
1\end{array}$ & $\begin{array}{l}8,4,2 \\
8,4,2\end{array}$ \\
\hline & $\mathrm{O}$ & $\begin{array}{l}1 \\
2\end{array}$ & $\begin{array}{l}1 \\
1\end{array}$ & $\begin{array}{l}16,8,4,2 \\
8,4,2\end{array}$ \\
\hline
\end{tabular}

${ }^{\mathrm{a}}$ See Eq. (10) in the text.

(i) maximum partial wave in the expansion of the occupied orbitals in the direct potential $=58$,

(ii) maximum partial wave in the expansion of the occupied orbitals in the exchange potential $=40$ (oxygen $K$ shell), 40 (terminal nitrogen $K$ shell), 10 (central nitrogen

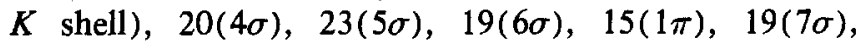
$15(2 \pi)$,

(iii) maximum partial wave in the expansion of $1 / r_{12}$ in the direct and exchange terms $=116$ and 58 , respectively,

(iv) all other partial wave expansions were truncated at $l=58$.

Based on our convergence studies of such expansions, this choice of expansion parameters should provide photoionization cross sections which are within a few percent of the converged values. The associated radial integrals were obtained using a Simpson's rule quadrature. The grid contained 750 points and extended out to 90.5 a.u. with a step size of 0.01 a.u. from the origin out to 3.5 a.u. Beyond 3.5 a.u. the largest step size was 0.4 a.u.

\section{RESULTS AND DISCUSSION}

Figure 1 shows our calculated $\sigma$ and $\pi$ contributions to the photoionization cross section for the $7 \sigma$ orbital of $\mathrm{N}_{2} \mathrm{O}$ leading to the $A^{2} \Sigma^{+}$state of $\mathrm{N}_{2} \mathrm{O}^{+}$along with the total cross section. The photon energy scale in this and other figures assumes the experimental ionization potential of 16.4 eV. ${ }^{13}$ These results were obtained using the dipole length form for the photoionization cross section. The cross sections obtained with the dipole velocity form for these cross sections differ only slightly from these results and are not shown in Fig. 1. Furthermore, these cross sections were calculated with the uniterated solutions of Eq. (8) for the photoelectron continuum orbitals. Calculations at several photon energies using more converged photoelectron orbitals

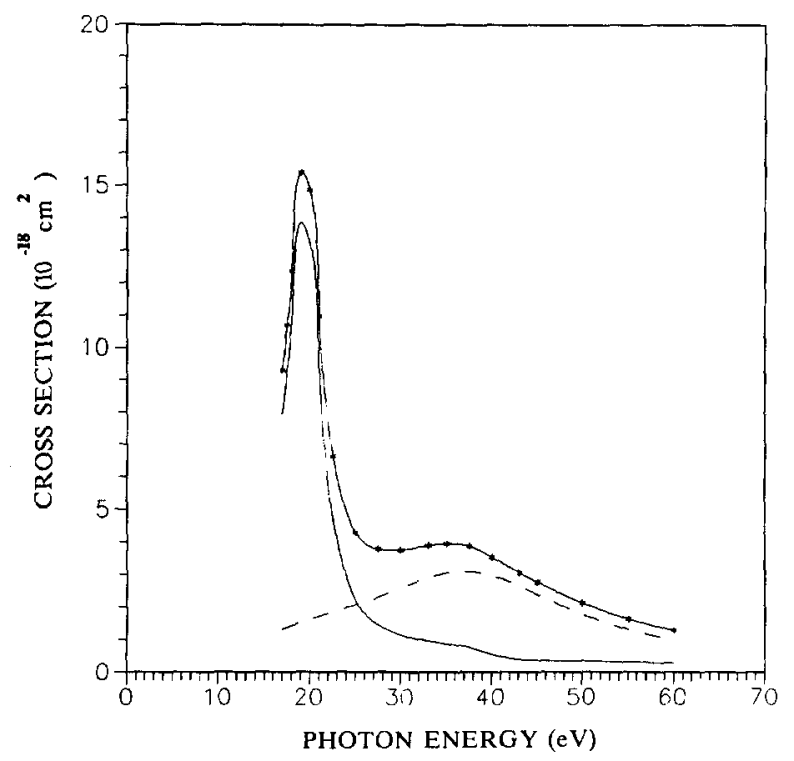

FIG. 1. Calculated photoionization cross sections for the $A^{2} \Sigma^{+}\left(7 \sigma^{-1}\right)$ state of $\mathrm{N}_{2} \mathrm{O}:-, 7 \sigma \rightarrow k \sigma$ component (dipole length); ---., $7 \sigma \rightarrow k \pi \mathrm{com}-$ ponent; - *-, total. Energies at which the cross sections were actually determined are indicated by *.

obtained with our iterative procedure for solving Eq. (6) ${ }^{17}$ show that the cross sections are, at worst, within $7 \%$ of the converged values.

The most significant feature in the cross sections of Fig. 1 is the prominent shape resonance in the $7 \sigma \rightarrow k \sigma$ channel just below $20 \mathrm{eV}$. The $7 \sigma \rightarrow k \pi$ contribution to the photoionization cross section is nonresonant and the broad enhancement around $38 \mathrm{eV}$ is due to the expected energy dependence of the dipole matrix element. Figure 2 shows the eigenphase sum for the $k \sigma$ continuum of this $\mathrm{N}_{2} \mathrm{O}^{+}\left(7 \sigma^{-1}\right)$ ion. The behavior of this eigenphase sum shows clearly that there are two resonances in this continuum. The low energy resonance associated with the increase in the eigenphase sum around 20 $\mathrm{eV}$ is very pronounced in the cross sections. However, the higher energy shape resonance, seen just below $40 \mathrm{eV}$ in the

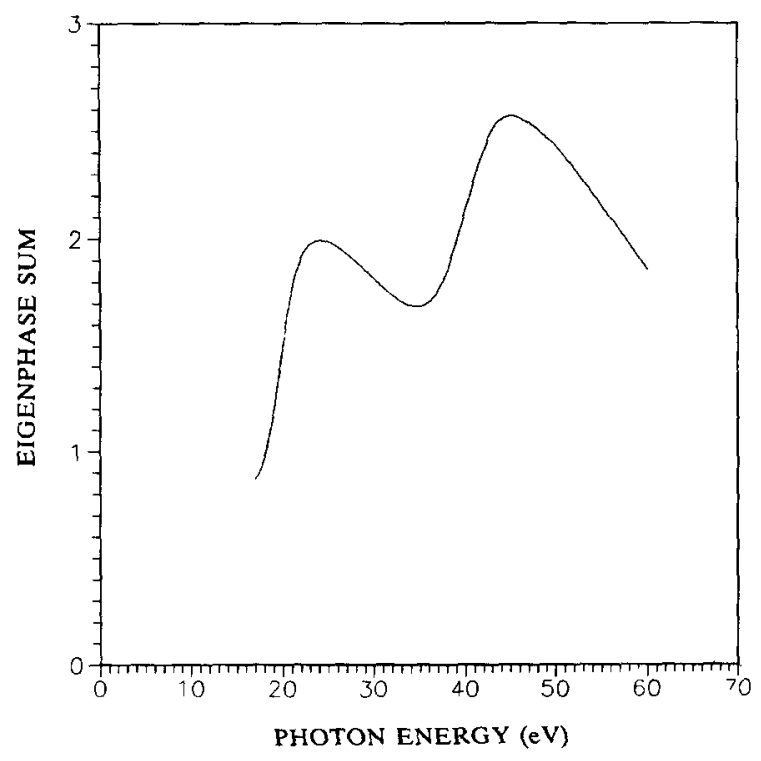

FIG. 2. Eigenphase sum for the $7 \sigma \rightarrow k \sigma$ channel in $\mathrm{N}_{2} \mathrm{O}$. 
eigenphase sum, is completely obscured in both the calculated and, as will be discussed, measured vibrationally unresolved photoionization cross sections. The composition of these resonances in terms of possible contributions from resonances associated with molecular subgroups, e.g., $\mathrm{N}-\mathrm{N}$ and $\mathrm{N}-\mathrm{O}$, will provide important physical insight into polyatomic shape resonances. Although the position of the low-energy shape resonance in $\mathrm{N}_{2} \mathrm{O}$ would be consistent with shifts expected on the basis of changes in NO bond distances between $\mathrm{NO}$ and $\mathrm{N}_{2} \mathrm{O}$, i.e., $2.173 a_{0}$ to $2.2418 a_{0}{ }^{16}$ the dependence of the eigenphase sum on the $\mathrm{N}-\mathrm{O}$ and $\mathrm{N}-\mathrm{N}$ distances in $\mathrm{N}_{2} \mathrm{O}$ shows that this resonance cannot be viewed as predominantly associated with the $\mathrm{N}-\mathrm{O}$ fragment. ${ }^{15}$ The behavior of these two shape resonances with changes in alternative vibrational modes, and combinations thereof, and the related vibrational branching ratios will be discussed in detail in a future publication. ${ }^{15}$

In Fig. 3 we compare our calculated cross sections with the experimental data of Truesdale et al..$^{13}$ and of Carlson et $a l .{ }^{12}$ The agreement between our cross sections calculated in the frozen-core Hartree-Fock fixed-nuclei approximation and the experimental data is quite good. This comparison clearly identifies the resonance feature around $20 \mathrm{eV}$ as due to a shape resonance. From these results it becomes clear that the slight enhancement of the cross sections around 35 $\mathrm{eV}$ is not shape resonant but comes from the usual energy dependence of the nonresonant $7 \sigma \rightarrow k \pi$ channel. Figure 3 also shows the cross sections obtained using the multiple scattering model with two different choices for the potential, i.e., the ground and transition state potentials. ${ }^{12}$ These results show a distinct shape resonant feature in the cross sections at 8 or $13 \mathrm{eV}$ photoelectron energy depending on the choice of potential. On this basis, Carlson et al. ${ }^{12}$ assigned the weak maximum around $32 \mathrm{eV}$ in the measured cross sections as due to a shape resonance. The present results show no such shape resonant enhancement of the vibration-

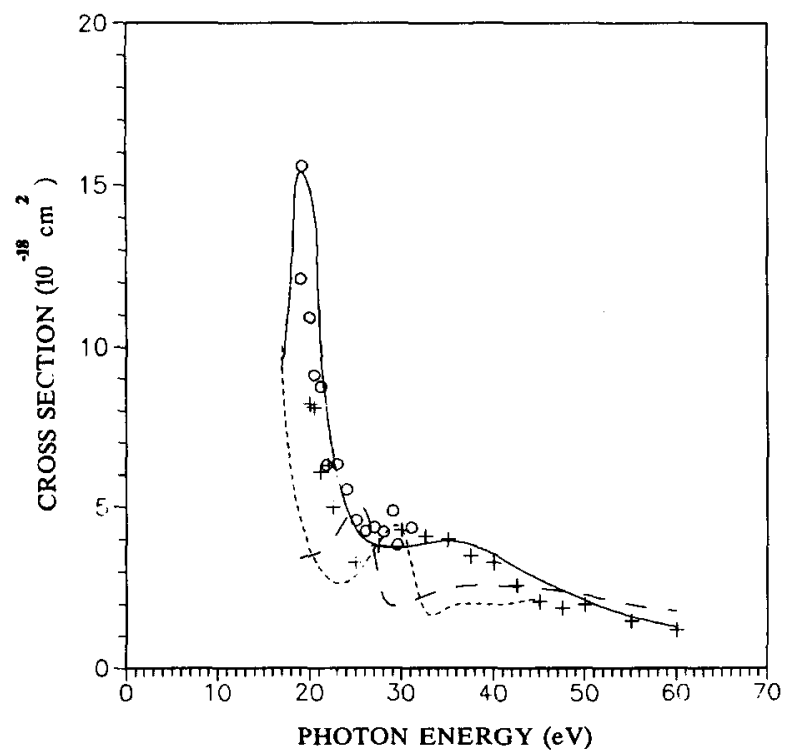

FIG. 3. Photoionization cross sections for the $A^{2} \Sigma^{+}\left(7 \sigma^{-1}\right)$ state of $\mathrm{N}_{2} \mathrm{O}$ : -, present results; $\mathrm{O}$, experimental data of Ref. 13 ; + , experimental data of Ref. 12; --., multiple scattering results (ground state potential) of Ref. 12; --- , multiple scattering results (transition state potential) of Ref. 12. ally unresolved cross sections in this energy range. Furthermore, on the basis of these multiple scattering results the rapid increase in the cross sections at lower photoelectron energy was not interpreted as arising from the low-energy shape resonance seen in the present Hartree-Fock results at a photoelectron energy around $3 \mathrm{eV}$. In fact, in the studies of Carlson et al. ${ }^{12}$ this low-energy resonance feature was attributed to autoionization. Although autoionization is known to occur in this spectrum below $20 \mathrm{eV},{ }^{22}$ our results show that a shape resonance must play a significant role in photoionization out of this $7 \sigma$ level at low photoelectron energy. In summary, the role of shape resonances in these photoionization spectra emerging from the present studies is quite different from that seen in the earlier multiple scattering results. ${ }^{12}$

In Fig. 4 we compare our calculated photoelectron asymmetry parameters for the $7 \sigma$ level of $\mathrm{N}_{2} \mathrm{O}$ with the measured values of Carlson et al. ${ }^{12}$ and of Truesdale et al..$^{13}$ The pronounced minimum at around $19 \mathrm{eV}$ in the calculated asymmetry parameters is obviously associated with the lowenergy shape resonance seen earlier in both the cross sections and eigenphase sums of Figs. 1 and 2, respectively. The agreement between the calculated and measured photoelectron asymmetry parameters for photon energies up to about $30 \mathrm{eV}$ is excellent. This agreement establishes that the rapid increase in the asymmetry parameters for photon energies above $19 \mathrm{eV}$ seen in the experimental data is due to a shape resonance. Our calculated asymmetry parameters show a second and broader minimum around $40 \mathrm{eV}$ arising from the higher energy shape resonance seen at about the same energy in the eigenphase sums of Fig. 2. It is important to note that this shape resonance was not seen in the vibrationally unresolved cross sections of Figs. 1 and 3. This broad minimum in the calculated asymmetry parameters around $40 \mathrm{eV}$ is in qualitative agreement with the somewhat more pronounced

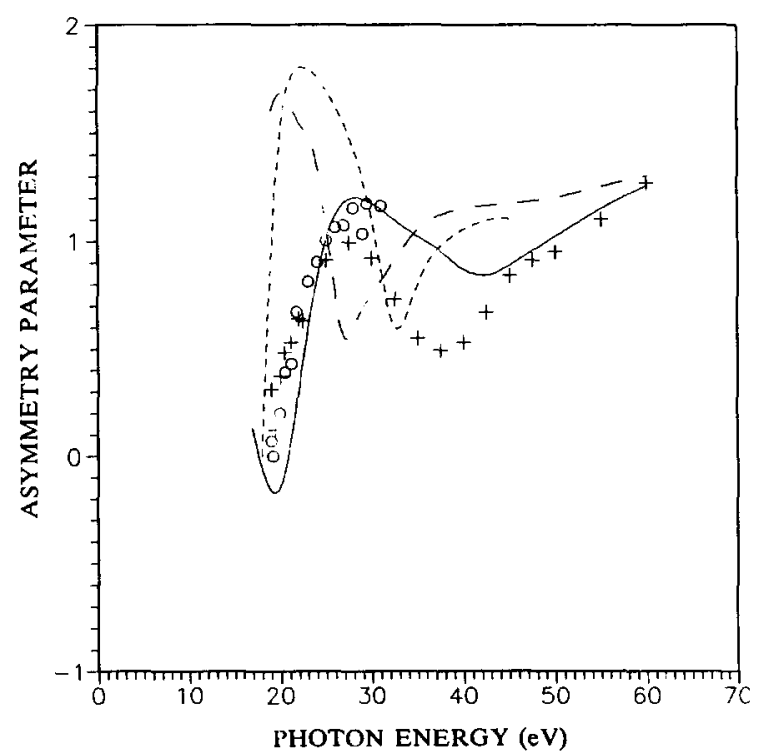

FIG. 4. Photoelectron asymmetry parameter for the $A^{2} \Sigma^{+}\left(7 \sigma^{-1}\right)$ state of $\mathrm{N}_{2} \mathrm{O}^{+}:-$, present results (dipole length); $\mathrm{O}$, experimental data of Ref. 13 ; + , experimental data of Ref. 12 ; -.-, multiple scattering results (ground state potential) of Ref. 12, - - , multiple scattering results (transition state potential) of Ref. 12 . 
minimum seen in the experimental data at $38 \mathrm{eV}$. Measurements of the vibrationally-resolved photoelectron spectra around this minimum would clearly help to identify possible reasons for the differences seen in the calculated and measured asymmetry parameters between 30 and $45 \mathrm{eV}$. Note that the data of Carlson et al. ${ }^{12}$ falls off more rapidly than that of Truesdale e $\mathrm{al}^{13}$ just above $30 \mathrm{eV}$. Finally, the calculated and measured asymmetry parameters agree very well beyond $45 \mathrm{eV}$.

Figure 4 also shows the photoelectron asymmetry parameters obtained using the multiple scattering model with different choices of the potential, i.e., the ground and transition potentials. ${ }^{12}$ These multiple scattering asymmetry parameters are substantially different from the present results calculated with frozen-core Hartree-Fock photoelectron orbitals. The differences between the multiple scattering and Hartree-Fock results for this polyatomic system are larger than generally seen previously in diatomic molecules. ${ }^{1}$ The multiple scattering results, particularly those using the ground state potential, do, however, show the presence of two shape resonances in these photoionization spectra.

\section{CONCLUDING REMARKS}

In this paper we have presented the cross sections and photoelectron asymmetry parameters for photoionization of the $7 \sigma$ level of $\mathrm{N}_{2} \mathrm{O}$. These studies were motivated by recent dispersed fluorescence measurements ${ }^{14}$ which showed significant non-Franck-Condon behavior in the vibrational branching ratios for both the symmetric and asymmetric stretching modes at low photoelectron energies where previously only autoionizing resonances, but no shape resonance, had been identified. ${ }^{12,13}$ Earlier vibrationally unresolved measurements of these cross sections had been interpreted, partly on the basis of multiple scattering studies, in terms of autoionizing resonances at low photoelectron energies and one shape resonance at considerably higher energy in the $k \sigma$ channel. Our results establish that there are two $\sigma$ shape resonances in the $7 \sigma$ photoionization of $\mathrm{N}_{2} \mathrm{O}$. One is a low-energy resonance which is very evident in both the cross sections and asymmetry parameters. The other occurs at higher energy where it is essentially obscured in the vibrationally unresolved cross sections but is clearly seen in the asymmetry parameters. Although unusual, certainly for diatomic molecules, the presence of two shape resonances in the same continuum as seen here promises to be more common for polyatomics. ${ }^{7}$ Studies of the vibrationally resolved cross sections in the region of these shape resonances which are in progress reveal a rich and unusual dependence of these resonances on changes in internuclear distance. ${ }^{15}$ Such studies along with experimental measurements of vibrational branching ratios using dispersed fluorescence techniques can be expected to provide much needed insight into the nature of resonant photoionization in polyatomic molecules.

\section{ACKNOWLEDGMENTS}

This material is based upon research supported by the National Science Foundation under Grant No. CHE8521391. The authors also acknowledge use of the resources of the San Diego SuperComputer Center which is supported by the National Science Foundation.

'J. L. Dehmer, D. Dill, and A. C. Parr, in Photophysics and Photochemistry in the Vacuum Ultraviolet, edited by S. McGlynn, G. Findley, and R. Huebner (Reidel, Dordrecht, 1985), pp. 341-408.

${ }^{2}$ See, for example, J. L. Dehmer, A. C. Parr, and S. H. Southworth, in Handbook on Synchrotron Radiation, edited by G. V. Marr (North-Holland, Amsterdam, 1986), Vol. II.

${ }^{3}$ T. A. Carlson, M. O. Krause, F. A. Grimm, J. D. Allen, D. Mehaffy, P. R. Keller, and J. W. Taylor, Phys. Rev. A 23, 3316 (1981).

${ }^{4}$ R. P. Lucchese and V. McKoy, Phys. Rev. A 26, 1406 (1982).

${ }^{5}$ D. M. P. Holland, A. C. Parr, D. L. Ederer, J. B. West, and J. L. Dehmer, Int. J. Mass. Spectrom. Ion Phys. 52, 195 (1983).

${ }^{6} \mathrm{~J}$. Kreile, A. Schweig, and W. Thiel, Chem. Phys. Lett. 100, 351 (1983).

'D. L. Lynch, S. N. Dixit, and V. McKoy, J. Chem. Phys. 84, 5504 (1986).

${ }^{8}$ J. B. West, A. C. Parr, B. E. Cole, D. L. Ederer, R. Stockbauer, and J. L. Dehmer, J. Phys. B 13, L105 (1980).

${ }^{9}$ R. Stockbauer, B. E. Cole, D. L. Ederer, J. B. West, A. C. Parr, and J. L. Dehmer, Phys. Rev. Lett. 43, 757 (1979).

${ }^{10}$ B. E. Cole, D. L. Ederer, R. Stockbauer, K. Codling, A. C. Parr, J. B. West, E. D. Poliakoff, and J. L. Dehmer, J. Chem. Phys. 72, 6308 (1980).

"See, for example, P. Roy, I. Nenner, M. Y. Adam, J. Delwiche, M. J. Hubin-Franskin, P. Lablanquie, and D. Roy, Chem. Phys. Lett. 109, 607 (1984).

${ }^{12}$ T. A. Carlson, P. R. Keller, J. W. Taylor, T. Whitley, and F. Grimm, J. Chem. Phys. 79, 97 (1983).

${ }^{13}$ C. M. Truesdale, S. Southworth, P. H. Kobrin, D. W. Lindle, and D. A. Shirley, J. Chem. Phys. 78, 7117 (1983).

${ }^{14}$ E. D. Poliakoff, M.-H. Ho, M. G. White, and G. E. Leroi, Chem. Phys. Lett. 130, 91 (1986)

${ }^{15} \mathrm{M}$. Braunstein and V. McKoy, J. Chem. Phys. (to be published)

${ }^{16}$ T. Gustafsson and H. J. Levinson, Chem. Phys. Lett. 78, 28 (1981).

${ }^{17}$ R. R. Lucchese and V. McKoy, Phys. Rev. A 24, 770 (1981).

${ }^{18}$ See, for example, T. H. Dunning, Jr. and P. J. Hay, in Methods of Electronic Structure Theory, edited by H. F. Schaefer III (Plenum, New York, 1977).

${ }^{19}$ R. R. Lucchese and V. McKoy, Phys. Rev. A 26, 1406 (1982)

${ }^{20}$ R. R. Lucchese and V. McKoy, Phys. Rev. A 28, 1382 (1983).

${ }^{21}$ A. D. McLean and M. Yoshimine, Tables of Linear Molecular Wave Functions (IBM Research Laboratory, San Jose, 1967), p. 200.

${ }^{22}$ See, for example, E. D. Poliakoff, M.-H. Ho, G. E. Leroi, and M. G. White, J. Chem. Phys. 85, 5529 (1986). 